\title{
THREE-DIMENSIONAL MODELLING AND SIMULATION THEORY OF FIELD EFFECT DEVICES
}

\author{
M. N. DOJA ${ }^{\mathrm{a}, *}$, MOINUDDIN $^{\mathrm{b}}$ and UMESH KUMAR ${ }^{\mathrm{b}}$ \\ ${ }^{a}$ Department of Electrical Engineering, Jamiamillia Islamia, India; \\ ${ }^{\mathrm{b}}$ IIT Delhi, India
}

(Received 30 October 1998; In final form 14 December 1998)

\begin{abstract}
In recent years, semiconductor manufacturers have been able to steadily reduce the physical area required by devices, thus allowing an increasing number of circuit functions on a single chip. Smaller semiconductor devices require more detailed and accurate analysis because minor errors can degrade their performance substantially.

A new, completely numerical three-dimensional model of a MOSFET allows a unified treatment of small devices. Preliminary results show that device size effects the surface potential and threshold voltage. Classical theory does not predict these effects. Although the model currently converges slowly, it can still be useful in analysing new small geometry devices.
\end{abstract}

Keywords: Modelling; simulation; FET

\section{INTRODUCTION}

The field effect transistor is a semiconductor device which depends for its operation on the control of current by an electric field. Its operation is based on the flow of majority carriers only. It is therefore a unipolar device. There are two types of field effect transistors the junction field effect transistor (JFET) and the insulated gate field effect transistor (IGFET) which is more commonly called the metal oxide semiconductor FET (MOSFET).

*Address for correspondence: C-46 Eastend Apartments, Mayur Vihar Phase 1 Ext., Delhi 110096, India. Tel.: 091-11-2717428, Fax: 6311261, e-mail: doja_el@jmi.ernet.in/ ndoja@hotmail.com 
The importance of the FET is a consequence of its properties. The first is its physical size; the MOSFET is so small compared to the BJT that it occupies only 20 to 30 per cent of the chip area taken up by a BJT. Thus MOSFETs can be packed quite densely on an IC chip and they are widely used for large scale integration (LSI). The second property is their extremely high input resistance. This means that the time constant of the input circuit is long enough to enable the charge stored on the small capacitance to remain sufficiently long for the device to be useful as a storage element in digital circuits. Another important property is that over a portion of their operating range they act like voltage controlled resistance elements and occupy much less area on the chip than the corresponding IC resistor. Also, MOSFETs can dissipate high power and switch large current is several nanoseconds; this enables the FET to be used as a high frequency high power switch.

Simple classical or regional approximation models and a completely numerical model are some of the options available for analysing the behaviour of semi conductor device before fabrication. A numerical model is preferred over a simple classical or regional approximation one. Numerical techniques can generate exact solutions of the most general character to simulate the semi conductor device. The exact model allows for arbitrary impurity distributions, recombination laws, mobility dependencies, injection levels and boundary conditions.

The number of dimensions one must use to describe the behaviour of a semi conductor device depends on the geometry of the structure and the type of device. One of the widely used semi conductor device is the MOSFET. Normally MOSFETs are simulated with two dimensional models which assume no variation of properties in the width direction. The assumption is only valid when the width is much greater than the depth of the channel depletion region, typically $0.5 \mu \mathrm{m}$. As the dimensions of the device are reduced, variations of properties along with the width as well as coupling with the length become significant. A MOSFET is considered small when its length and width have been shrunk to within an order of magnitude of the depth of the depletion region. For such small geometry MOSFETs, the third dimension has an important role in the overall device performance. Therefore, a general model must include this dimension. 


\section{VARIOUS TWO DIMENSIONAL APPROACHES}

To obtain general and exact analytical solutions for the MOSFET problem we require the solution of a system of non linear partial differential equations involving the Poisson equation, the majority carrier transport equation and the current continuity equation. But exact analytical solutions are improbable. As an alternative approach, several numerical solutions have been published.

De la Moneda used a finite difference method for solving the two dimensional Poisson equation [1]. But this method is an extension of the old model to take into account the two dimensional nature of the electrostatic potential, and is based on that developed by Kim and Yang in their JFET analysis [2].

Scott and Chamberlain have used a regional charge density approximation in the solution of Poisson's equation, and derived an analytical solution of the continuity equation. This model can be used for the estimation of subthreshold leakage current in MOSFET dynamic random access memories.

Poon has suggested a compact four parameter fitting, model that can be applied over a wide range of currents and channel lengths.

Narrow width devices exhibit an increase in threshold voltage. This effect becomes more pronounced as the device width becomes smaller. To calculate this effect, a two dimensional numerical analysis of Buturula and Cettrell can be employed.

\section{THREE DIMENSIONAL MODELLING}

\section{a. Its Requirement}

The device structure is essentially three dimensional. When channel length and/or width is large, this three dimensional problem can be reduced to a two dimensional one. However, with the advent of VLSI where MOSFETs of sub micrometer channel lengths and widths are called for, device simulation at the three dimensional level has become a must.

Most investigators have focussed on two dimensional models to account for short channel effects. But Kothecha and Bellistein have 
pointed out that three dimensional effects should be taken into account for more representative current and capacitive models. It is only with a three dimensional device simulator that the mechanism whereby narrow channel effects occur has been first explained adequately.

Considerable differences exist between two and three dimensional analysis. The differences becomes more pronounced as the device size gets reduced. Therefore, three dimensional models are preferable for the analysis of small geometry devices.

\section{b. Various Three Dimensional Attempts}

Shima, Sugwara and Moriyana made a table look up MOSFET model for circuit simulation [11]. A major problem is that it requires a massive storage capacity for three dimensional table structure.

Accurate three dimensional analysis of semi conductor devices based on general transport equation is given by Toshii, Sudo et al. They used the finite difference scheme for numerical analysis; formulation to reduce time and memory requirements; and relaxation method to help solve a large size matrix.

Besides these, threshold voltage analysis of short and narrow channel MOSFETs by three dimensional computer simulation is given by Kasai, Sudo et al. [7]. Here sufficient convergence rate with acceptable memory size is obtained from optimum mode discretization and initial solution guess with sophisticated boundary conditions.

\section{THE NOVEL THREE DIMENSIONAL MODEL}

Symbols and their normalizing constants

\begin{tabular}{lcc}
\hline Symbol & Name of variable & $\begin{array}{c}\text { Normalizing } \\
\text { constant }\end{array}$ \\
\hline$N_{A}$ & Doping density & $n_{i}$ \\
$E$ & Electric field & $k T / q L_{D}$ \\
$\mathrm{k}$ & Boltzmann's constant & \\
$L$ & Channel length & $L_{D}$ \\
$m$ & Iteration number & $n_{i}$ \\
$n, p, n_{i}$ & Electron, hole and intrinsic carrier density & $n_{i}$ \\
$N^{+}$ & Source and drain doped regions & \\
$q$ & Electron charge & \\
$T$ & Absolute temperature & \\
\hline
\end{tabular}


Symbols and their normalizing constants (Continued)

\begin{tabular}{lcc}
\hline Symbol & Name of variable & $\begin{array}{c}\text { Normalizing } \\
\text { constant }\end{array}$ \\
\hline$V_{s}, V_{D}, V_{G}, V_{B G}$ & Source, drain, gate and backgate voltage & $k T / q$ \\
$x, y, z$ & Space coordinates & $L_{D}$ \\
$\nabla$ & Gradient operator & \\
$\nabla^{2}$ & Laplacian operator & \\
$\varepsilon_{s}, \varepsilon_{d}$ & Permittivity of silicon and $\mathrm{SiO}_{2}$ insulator \\
$\varepsilon_{1}$ & material & $k T / q$ \\
$\delta$ & Small error margin & $k T / q$ \\
$\varphi_{n}, \varphi_{p}$ & Difference in electrostatic potential & $k T / q$ \\
$\Psi$ & Electron and hole quasi-Fermi potentials & \\
$w$ & Electrostatic potential & \\
\hline
\end{tabular}

\section{a. Lindholm-Hamilton Theory}

Before proceeding with the development of the modeling theory, we briefly discuss the theory of Lindholm and Hamilton. Models obtained by using Lindholm and Hamilton's techniques are derived systematically. The models incorporate a direct relationship to device morphology and the physical processes. Furthermore, models of variable complexity and accuracy can be developed.

The theory of Lindholm and Hamilton contains some limitations which must be removed. The entire theory is based on the assumption of one directional current flow. Also, the theory does not incorporate non linear effects. Indeed, even some linear effects e.g., surface recombination, are not related to the modelling.

The theory suggests the use of four distinct approaches i.e., approximating techniques to the modelling problem. When the model is extended to three dimensions, it is convenient to use a combination of approximating techniques. The fundamental approximation used here is the quasi static approximation i.e., the use of steady state information in the solution of a time varying problem. The assumption involved here is that relations among various parameters e.g., current components and carrier densities, under steady state (DC) conditions prevail under time varying conditions as well.

\section{b. Boundary Conditions}

In modelling theory, we must describe the relations existing between the terminal currents and terminal voltages. Therefore, we must 
supplement the model of the bulk region with the model of the surrounding surface.

Generally, the surfaces subject to external constraints are boundaries of depletion regions of $p$ - $n$ junctions i.e., a forward biased junction would result in non-zero independent boundary conditions on the minority carrier densities on either side of the junction. Hence, the model must be supplemented in this case with $p-n$ junction models as well as other pertinent information.

\section{MODEL DEVELOPMENT}

The three dimensional steady state model of a MOSFET consists of a set of coupled, non linear partial differential equations. The complete set of equations include Poisson and Laplace's equations, the majority and minority continuity equations, the transport equations and auxiliary equations describing mobility, recombination and carrier densities.

One objective of the model was to investigate the effects of geometry on the threshold voltage (the voltage required to turn the device on). The current is very low at this voltage level and its effect on the field can be neglected. Thus we can remove the transport and continuity equations from the system equations. We need then develop only an electrostatic model.

Figures 1 and 2 show the geometry of the model of the MOSFET used here. The device consists of a $p$ type semi conductor substrate and two $\mathrm{N}^{+}$regions formed by either diffusion or ion implantation. The upper surface of the device is covered with an insulator $\left(\mathrm{SiO}_{2}\right)$ which is covered by an electrode. The electrode may be aluminum (A1), N Polysilicon, P Polysilicon, molybdenum dislicide $\left(\mathrm{MoSi}_{2}\right)$, or other materials as long as the work function is defined.

We use Cartesian coordinates to describe the model. The horizontal coordinate $x$ is parallel to the surface insulator. The horizontal original is the left edge of the device and the axis is positive in the right direction. The vertical coordinate $y$ is normal to the insulator interfaces. The vertical origin is the top of the $\mathrm{SiO}_{2}$ insulator and the axis is positive in the direction of the substrate. The width direction $z$ is positive into the device.

The MOSFET is assumed to be structurally perfect. Deliberately introduced impurity densities are not considered defects. Lattice 


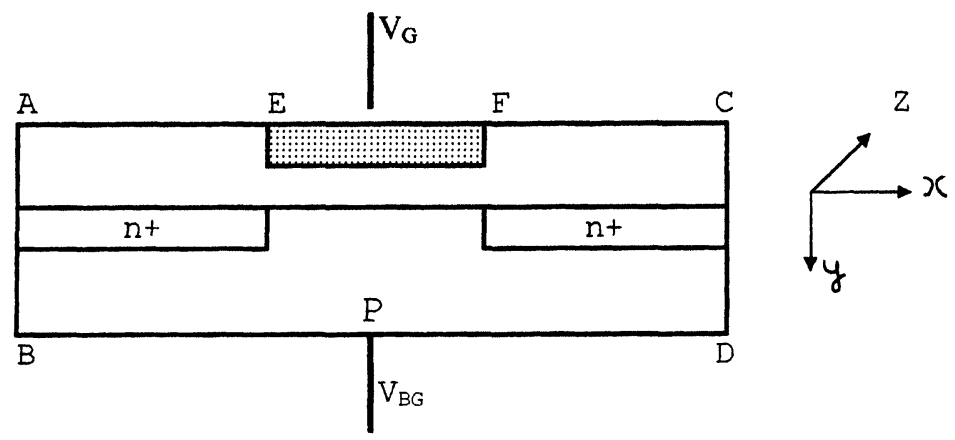

FIGURE 1 Length cross section of a MOSFET.

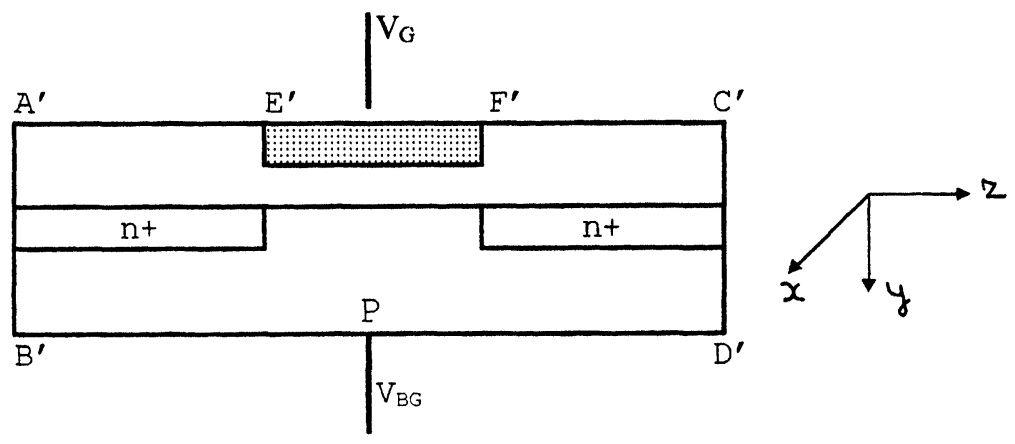

FIGURE 2 Width cross section of a MOSFET.

strains and dislocations introduced during the diffusion of high concentrations of impurities are neglected.

We must make several assumption and approximations to make the calculations manageable. All of these which are given below are reasonable under normal operating conditions.

1. Ideal contacts and charge neutrality at the source and drain.

2. The impurity dopant is completely ionized and its distribution remains constant in time.

3. Non degenerate doping levels to allow the use of Boltzmann statistics for calculating hole and electron concentrations.

4. Isothermal spatial conditions. 
The electrical behavior of the semi conductor device can be described by Poisson's and Laplace's equations. Solving this set of non linear partial differential equations with appropriate boundary conditions gives us the potential distribution and the carrier concentrations.

The Poisson equation can be expressed as

$$
\begin{aligned}
& \frac{d^{2} \Psi(x, y, z)}{d x^{2}}+\frac{d^{2} \Psi(x, y, z)}{d y^{2}}+\frac{d^{2} \Psi(x, y, z)}{d z^{2}} \\
& =\frac{q}{\varepsilon_{s}}\left[P(x, y, z)+n(x, y, z)+N_{A}(x, y, z)\right]
\end{aligned}
$$

where

$\Psi$ is the electrostatic potential

$q$ is the electron charge

$\varepsilon_{s}$ is the permitivity of silicon

$p$ is the hole carrier density

$n$ is the electron density and

$N_{A}$ is the doping profile.

Laplace's equation is expressed as Poisson's equation with the right hand side equal to zero.

The carrier concentration can be obtained from the Boltzmann approximation of Fermi statistics as

$$
\begin{aligned}
& n(x, y, z)=n_{i} \exp \left\{(q / \mathrm{k} T)\left[N_{A}(x, y, z)-n(x, y, z)\right]\right\} \\
& p(x, y, z)=n_{i} \exp \left\{(q / \mathrm{k} T)\left[p(x, y, z)-N_{A}(x, y, z)\right]\right\}
\end{aligned}
$$

where

$n$ is the electron quasi Fermi potential

$p$ is the hole quasi Fermi potential

$n_{i}$ is the intrinsic carrier concentration

$\mathrm{k}$ is Boltzmann's constant and

$T$ is the absolute temperature.

The semiconductor bulk impurity distribution $N_{A}(x, y, z)$ may be described by several methods. A uniform impurity distribution, an 
analytic function or output files from a process fabrication program are all acceptable.

For mathematical convenience as well as numerical efficiency, we have normalized all variables. The normalization factors are in the list of Symbols and their normalizing equations will be listed, but it should be noted that the equations are not in their final form. The numerical approximations and solution methods determine the best form of the equations.

$$
\begin{gathered}
\nabla^{2} \Psi=n-p+N_{A} \\
\nabla^{2} \Psi=0 \\
n=\exp \left(\Psi-\phi_{n}\right) \\
p=\exp \left(\phi_{p}-\Psi\right)
\end{gathered}
$$

We must also determine appropriate boundary conditions for the device of Figure 1. Using the labelling of this figure, we have:

$$
\begin{gathered}
\text { on } \quad \mathrm{AB}, \mathrm{CD} \quad \frac{\partial \Psi}{\partial x}=0 \\
\text { and on } \mathrm{AE}, \mathrm{FC} \quad \frac{\partial \Psi}{\partial y}=0
\end{gathered}
$$

Using Figure 2 we have

$$
\text { on } \quad \mathrm{A}^{\prime} \mathrm{B}^{\prime}, \mathrm{C}^{\prime} \mathrm{D}^{\prime} \quad \frac{\partial \Psi}{\partial z}=0
$$

On the contacts the applied voltage is known. Since the space charge density is zero, the quasi-Fermi potentials can be determined. Therefore,

1. on the source contact:

$$
\Psi_{s}=\frac{\mathrm{k} T}{q} \ln \left[\frac{N^{+} N_{A}}{n_{i}^{2}}+V_{s}+V_{B G}\right]
$$




$$
\phi_{n}=\phi_{p}=\ln \left[\frac{N_{A}}{n_{i}}\right]
$$

2. on the drain contact:

$$
\begin{gathered}
\Psi_{D}=\frac{\mathrm{k} T}{q} \ln \left[\frac{N^{+} N_{A}}{n_{i}^{2}}+V_{D S}+V_{B G}\right] \\
\phi_{n}=\phi_{p}=\ln \left[\frac{N^{+} N_{A}}{n_{i}}\right]
\end{gathered}
$$

3. on the substrate contact:

$$
\Psi_{B G}=0
$$

The backgate of the substrate is taken as the zero level for the electrostatic potential. Therefore, we must add the built in voltage between the source and substrate and the drain and substrate to the source and drain potential.

4. on the gate contact:

$$
\Psi_{g}=\frac{q V_{g}}{\mathrm{k} T}
$$

on the substrate-oxide interface

a. $\Psi$ is continuous

b. $(\partial \Psi / \partial y)$ is discontinuous by the amount of fixed interface charge.

The elliptical Eqs. (4) and (7) together with the mixed boundary conditions (8) to (18) determine the steady state behaviour of the MOSFET.

The first step is solving the system equations numerically is to discretize the spatial variables. Either finite difference or element methods could be used. We choose the finite difference method. We would like to know whether a unique solution exists, but this is difficult to demonstrate for a MOSFET. In this work, we assume the existence and uniqueness of the solution. Previous results justify this assumption. 
We define

$$
\begin{gathered}
\gamma_{n}=\exp \left(-\phi_{n}\right) \\
\gamma_{p}=\exp \left(-\phi_{p}\right)
\end{gathered}
$$

So that we may write Poisson's equation in the semi conductor as

$$
\nabla^{2} \Psi=\gamma_{n} e^{\Psi}-\gamma_{p} e^{-\Psi}+N_{A}
$$

and Laplace's equation in the insulator as

$$
\nabla^{2} \Psi=0
$$

The solution of Poisson's equation requires the initialization of $\Psi$, $\phi_{n}$ and $\phi_{p}$. The most common approach to solving Poisson's equation is a quasi linearization method. Defining the solution of Eq. (4) as $\Psi_{\text {exact }}$ and the difference between $\Psi_{\text {exact }}$ and $\Psi_{\text {iterated }}$ as $\delta$ where

$$
\delta=\Psi_{\text {exact }}-\Psi_{\text {iterated }}
$$

Equation (4) now takes the form

$$
\nabla^{2} \Psi+\nabla^{2} \delta=\exp \left(\Psi+\delta-\phi_{n}\right)-\exp \left(\phi_{p}-\delta-\Psi\right)+N_{A}
$$

if $\delta \ll 1$ and $\exp ( \pm \delta)=1 \pm \delta$ then,

$$
\begin{aligned}
& \nabla^{2} \delta-\delta\left[\exp \left(\Psi-\phi_{n}\right)+\exp \left(\phi_{p}-\Psi\right)\right] \\
& \quad=\nabla^{2} \Psi+\exp \left(\Psi-\phi_{n}\right)-\exp \left(\phi_{p}-\Psi\right)+N_{A}
\end{aligned}
$$

Here $\Psi$ stands for $\Psi_{\text {iterated }}$.

The general form of a linear elliptical differential equation with constant coefficients can be expressed as

$$
\mathrm{AU}_{x x}+\mathrm{BU}_{y y}+\mathrm{CU}_{z z}+\mathrm{DU}_{x}+\mathrm{EU}_{y}+\mathrm{FY}_{z}+\mathrm{GU}+\mathrm{H}=\mathrm{O}
$$

with $\mathrm{A}>\mathrm{O}, \mathrm{B}>\mathrm{O}, \mathrm{C}>\mathrm{O}$ and $\mathrm{G}<=\mathrm{O}$.

We take $\mathrm{A}=1, \mathrm{~B}=1, \mathrm{C}=1, \mathrm{D}=\mathrm{E}=\mathrm{F}=\mathrm{O}$. 
The discrete analog for the general linear elliptic differential equation is

$$
\begin{aligned}
\alpha_{0} \Psi(i, j, k) & +\alpha_{1} \Psi(i+1, j, k)+\alpha_{2} \Psi(i, j+1, k)+\alpha_{3} \Psi(i, j, k+1) \\
& +\alpha_{4} \Psi(i-1, j, k)+\alpha_{5} \Psi(i, j-1, k)+\alpha_{6} \Psi(i, j, k-1)+H=0
\end{aligned}
$$

where

$$
\begin{aligned}
& \alpha_{0}=-2\left[\frac{1}{h^{2}}+\frac{2 k_{j-1}}{k_{j}\left(k_{j}+k_{j-1}\right)}+\frac{2 k_{j}}{k_{j-1}\left(k_{j}+k_{j-1}\right)}+\frac{2}{l^{2}}\right] \\
& \alpha_{1}=\frac{1}{h^{2}}, \alpha_{2}=\frac{2 k_{j-1}}{k_{j} k_{j-1}\left(k_{j}+k_{j-1}\right)}, \alpha_{3}=\frac{1}{l^{2}}, \alpha_{4}=\frac{1}{h^{2}}, \\
& \alpha_{5}=\frac{2 k_{j}}{k_{j} k_{j-1}\left(k_{j}+k_{j-1}\right)}, \alpha_{6}=\frac{1}{l^{2}}
\end{aligned}
$$

here $h, k$ and $l$ are defined as shown in Figure 3.

Solving for the potential at the $m$ th iteration gives

$$
\begin{aligned}
\alpha(i, j, k)^{m}= & -\frac{\alpha_{0}}{\alpha_{1}} \Psi(i+1, j, k)^{m-1}-\frac{\alpha_{2}}{\alpha_{0}} \Psi(i, j+1, k)^{m-1} \\
& -\frac{\alpha_{3}}{\alpha_{0}} \Psi(i, j, k+1)^{m-1}-\frac{\alpha_{4}}{\alpha_{0}} \Psi(i-1, j, k)^{m} \\
& -\frac{\alpha_{5}}{\alpha_{0}} \Psi(i, j-1, k)^{m}-\frac{\alpha_{6}}{\alpha_{0}} \Psi(i, j, k-1)^{m}-\frac{H}{\alpha_{0}}
\end{aligned}
$$

This expression includes the Gauss-Seidel scheme of solution ordering. The solution goes from $(i, j, k),(i+1, j, k),(i+2, j, k) \ldots \ldots(i, j+l, k)$

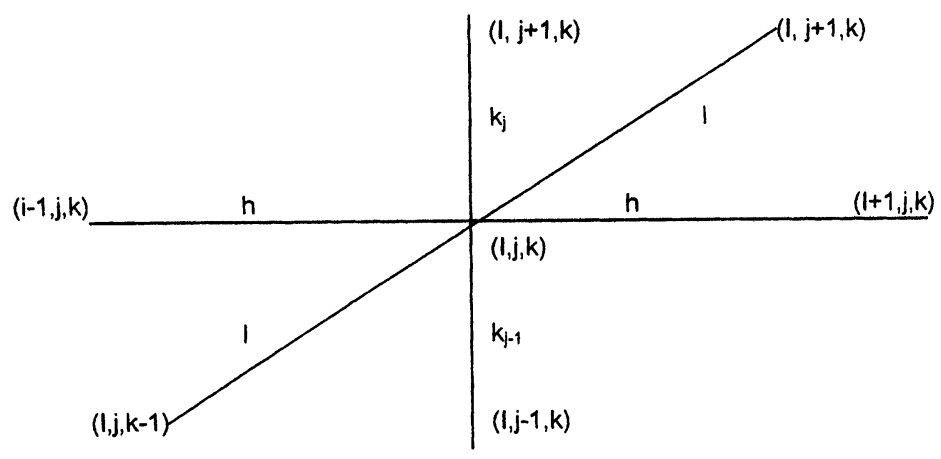

FIGURE 3 Mesh point notation. 
$(i+1, j+1, k)(i+2, j+1, k) \ldots(i, j, k+1),(i+l, j, k+1) \ldots$. The old values are replaced with the new solutions instantaneously instead of waiting for a row, block or complete matrix update.

We use a successive relaxation method with the following general form

$$
\Psi^{m}=\Psi^{m-1}+w\left(\Psi^{m}-\Psi^{m-1}\right)
$$

where when

$w<1$ under relaxation for potentially unstable solutions

$w=1$ direct substitution

$1<w<2$ over relaxation, for stable solutions and helps to speed convergence.

This optimum is normally impossible to determine analytically, and experimentation as well as experience is required. The complete expression for the potential in the silicon can be expressed as:

$$
\begin{aligned}
\Psi^{m}=(1-w) \Psi^{m-1}+w[ & -\frac{\alpha_{1}}{\alpha_{0}} \Psi(i+1, j, k)^{m-1}-\frac{\alpha_{2}}{\alpha_{0}} \Psi(i, j+1, k)^{m-1} \\
& -\frac{\alpha_{3}}{\alpha_{0}} \Psi(i, j, k+1)^{m-1}-\frac{\alpha_{4}}{\alpha_{0}} \Psi(i-1, j, k)^{m} \\
& \left.-\frac{\alpha_{5}}{\alpha_{0}} \Psi(i, j-1, k)^{m}-\frac{\alpha_{6}}{\alpha_{0}} \Psi(i, j, k-1)^{m}-\frac{H}{\alpha_{0}}\right]
\end{aligned}
$$

In the gate-oxide region, Laplace's equation must be solved. The coefficient reduce to

$$
\mathrm{D}=\mathrm{E}=\mathrm{F}=\mathrm{G}=\mathrm{H}=\mathrm{O}
$$

The potential is then

$$
\begin{aligned}
\Psi^{m}=(1-w) \Psi^{m-1}+w[ & -\frac{\alpha_{1}}{\alpha_{0}} \Psi(i+1, j, k)^{m-1}-\frac{\alpha_{2}}{\alpha_{0}} \Psi(i, j+1, k)^{m-1} \\
& -\frac{\alpha_{3}}{\alpha_{0}} \Psi(i, j, k+1)^{m-1}-\frac{\alpha_{4}}{\alpha_{0}} \Psi(i-1, j, k)^{m} \\
& \left.-\frac{\alpha_{5}}{\alpha_{0}} \Psi(i, j-1, k)^{m}-\frac{\alpha_{6}}{\alpha_{0}} \Psi(i, j, k-1)^{m}-\frac{H}{\alpha_{0}}\right]
\end{aligned}
$$




\section{THE ITERATIVE METHOD}

An initial state i.e., $\phi_{n}(i, j, z), \phi_{p}(x, j, z)$ is required to specify the device completely for the first iteration. This initial state can be a zero bias state or a steady state bias state. Experience shows that the initial guess is important. A good initial guess reduces the number of required iterations considerably whereas a poor initial guess can cause stability problems. We made extensive use of results from a two dimensional model to provide the initial guess. The initial distribution of the variables is then inserted into Poisson's or Laplace's equation and an improved $\Psi(i, j, k)^{m+1}$ results. The superscript $m$ is the space iteration number.

After obtaining an improved solution for the dependent variables, we check to see if a predetermined degree of consistency has been achieved in the iterative solution. For example, let

$$
\left|\Psi(i, j, k)^{m+1}-\Psi(i, j, k)^{m}\right|<\varepsilon_{1}
$$

where $\varepsilon_{1}$ is a small error such as $10^{-6}$. If the criterion stated in the Eq. (34) is met over the entire spatial grid, the iterative process has converged. If the desired degree of consistency has not been achieved, the iterative process continues until convergence is obtained.

\section{CONCLUSION}

A three dimensional model of a small geometry MOSFET has been described. The model calculates the internal distributions for the potential and the carrier density for various gate and drain voltages.

Numerical instabilities were eliminated by the proper choice of the relaxation factor, $w=1.2$. The convergence rate is very slow and has to be improved.

The model can be modified to include the effect of a tapered recessed oxide. This is important since most devices today have a tapered rather than as abrupt oxide transition region. This will require changing the semi conductor oxide interface from the surface to a depth of approximately 4000 and then stair stepping the interface to the surface. 


\section{References}

[1] De La Moneda F. H. (1973). Threshold voltage from numerical solution of the two dimensional MOS transistor, IEEE Trans., CT-20, 666-673.

[2] Kim, C. K. and Yang, E. S. (1970). An analysis of current saturation mechanism of junction field effect transistor, IEEE Trans., ED-17, 120-127.

[3] Wang, P. P. (1978). Devices characteristics of short channel and narrow width MOSFET's, IEEE Trans., ED-25, 779-786.

[4] Merckel, G. (1980). A simple model of the threshold voltage of short and narrow channel MOSFET's, Solid State Electronics, pp. 1207-1213.

[5] Yamaguchi, K. (1979). Field dependent mobility model for the two dimensional numerical analysis of MOSFET's, IEEE Trans., ED-26, 1068-1074.

[6] Masuda, H., Nakai, M. and Kubo, M. (1979). Characteristics and limitations of scaled down MOSFET's due to two dimensional field effect, IEEE Trans., ED-26, 980-986.

[7] Kasai, R., Tokoyoma, K., Yoshii, A. and Sudo, T. (1982). Threshold voltage analysis of short and narrow channel MOSFET's by three dimensional computer simulation, IEEE Trans., ED-29, 970-976.

[8] Yoshii, A., Kitazawa, H., Tomizawa, M., Horiguchi, S. and Sudo, T. A. (1982). Three dimensional analysis of semiconductor devices, IEEE Trans., ED-29, 184-189.

[9] Shigyo, N. and Konaka, Dang (Toshiba Corp. Kawasaki, Japan): 82-35803: Three dimensional simulation of inverse narrow channel effect.

[10] Kasai, R., Yokoyoma, K., Yoshii, A. and Sudo, T. (1982). (Electrical Comm. Labs., Nippon Telegraph and Telephone Public Corp. Tokyo, Japan): Threshold voltage analysis of short and narrow channel MOSFET's by three dimensional computer simulation, IEEE Trans. Electron Devices, ED-29, 870-876.

[11] Shina, T., Sugawara, T., Moriyana, S. and Yamada, H. (1982). (Toshiba, R. and Kawasaki, D.): 3-D Table look up MOSFET model for precise circuit simulation, IEEE J. Solid State Circuits, SC-17, 449-454.

[12] Akers, L. A. and Ta Ming, Wang (1983). (Dep. of Elec. and Comp. Engg., Arizona State University, USA): Simulation of a three dimensional semi conductor device, Simulation, 40(2), 43-50.

[13] Shoggo, N. (1985). Analysis of an anomalous subthreshold current in a fully accessed oxide MOSFET using 3-D device simulator, IEEE Trans., ED-32, 441-445. 

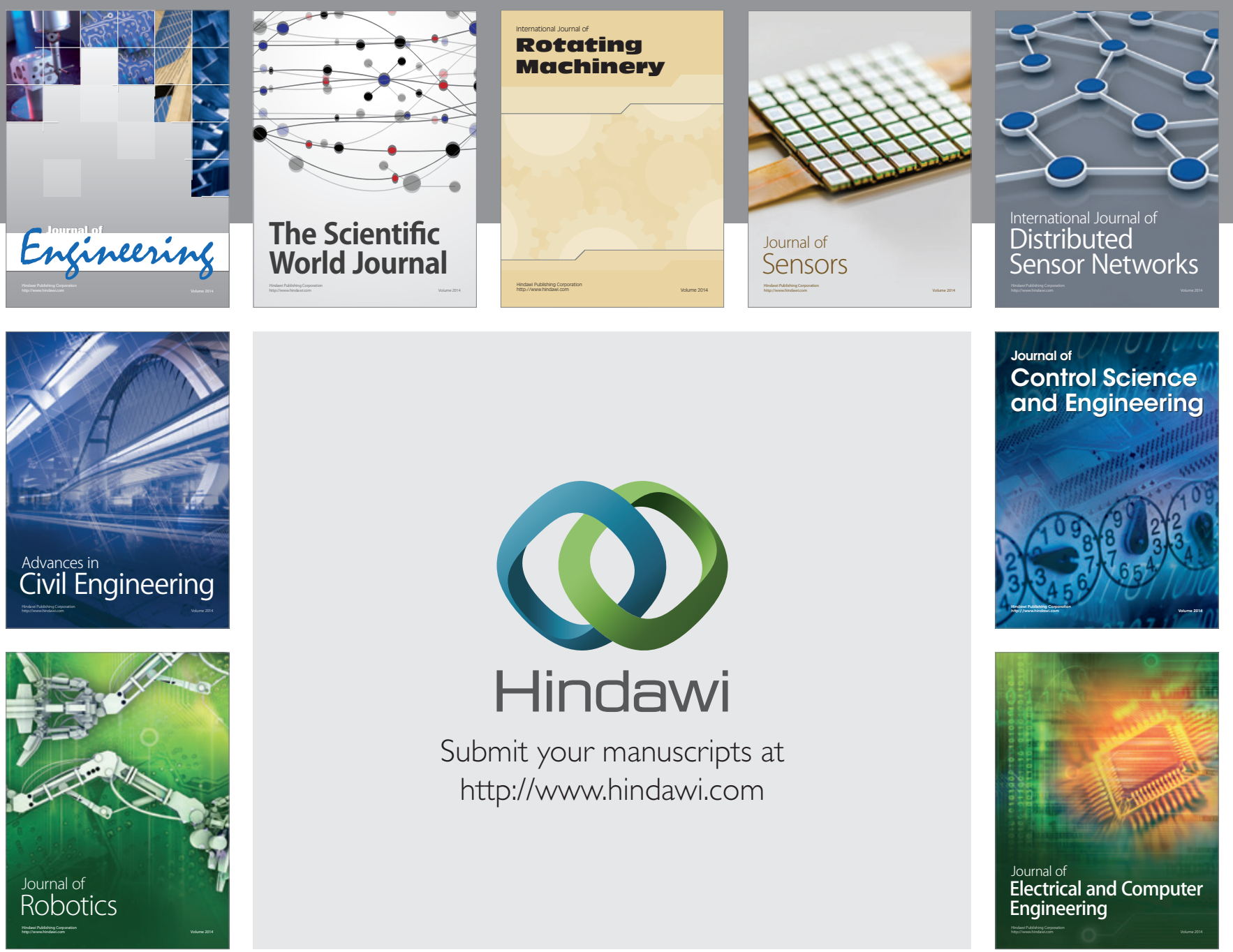

Submit your manuscripts at

http://www.hindawi.com
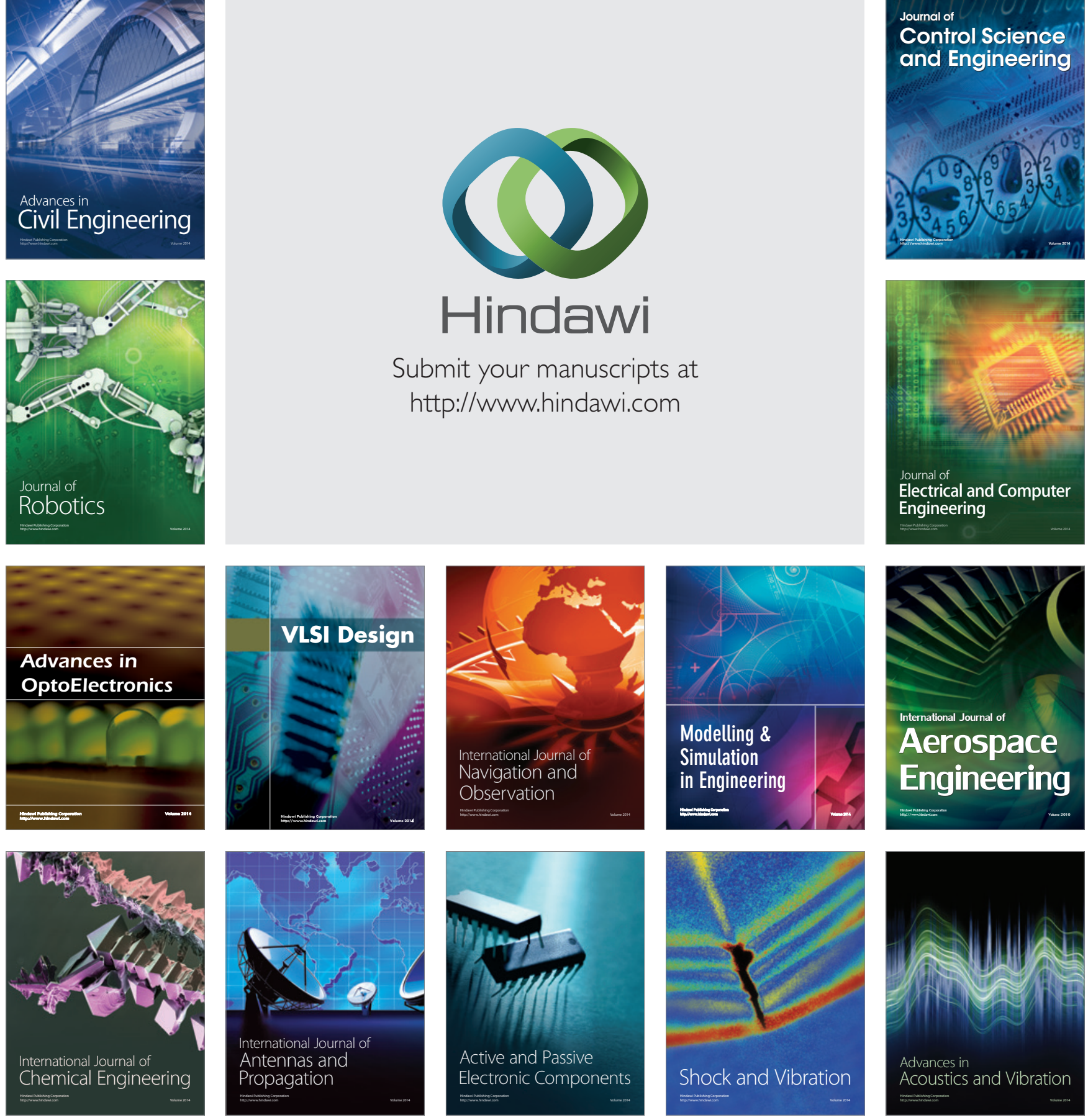